\title{
Development of a filter amplifier of the signal pre-processing device for the passive listening mode of the hydroacoustic complex (HAC)
}

\author{
D.A. Hviyuzova, A.A. Belitskiy \\ Saint Petersburg Mining University, Department of Electronic Systems, St Petersburg, Russia
}

\begin{abstract}
The article describes the process of developing a filter amplifier, which is part of the signal pre-processing device. The requirements to the filter, which must be obtained at the output of the developed circuit diagram, have been established. It is described what the main elements of the circuit are responsible for and how they have been selected. It is shown that the developed device can find application in sonar technology.
\end{abstract}

\section{Introduction}

Hydroacoustics as a means of obtaining information from the aquatic environment has found application in research of the oceans and in solving many applied problems associated with the operation of civil and military vessels. The main ones include the following features: ensuring safe navigation (measuring depth, speed, determining the fairway, etc.); detection of the military (surface and submarine ships, mines, etc.) and civilian (sunken ships and not only schools of fish, etc.) objects; search and extraction of mineral resources located on the ocean floor; etc.

The most complex systems are those that provide naval combat missions, namely: sonar stations (GAS), which solve a separate functional task, such as direction finding and echoing, mine search and torpedo detection; sonar systems (HAC), which are a complex multifunction system of receiving, processing, classifying, emitting sonar signals and displaying information about the underwater environment, with centralized control and subject to solving one single task. Hydroacoustic complexes, as a rule, include systems of passive and active direction finding, detection of hydroacoustic signals, communication, near-field overview, navigation systems, etc $[1,4,5]$.

The complexity and variety of factors affecting the conditions for the propagation of hydroacoustic signals, their inconsistency in space and time, new tasks, and growing requirements to increase the efficiency of hydroacoustic systems compel scientists and engineers to constantly work on the study of environmental characteristics, development and refinement models of signals and interference and the search for adequate and feasible signal processing algorithms. One of the factors determining the effectiveness of signal processing and affecting the achievement of certain characteristics of hydroacoustic systems is the level of development of electronics, which in our time allows us to perform such tasks in easily accessible ways. 
The most important task of the equipment designer, in addition to creating a workable circuit, at the moment is to ensure the normal operation of the equipment in the "real world" with the actual presence of other equipment near it. This means that sources of external noise should not affect the device, and it shouldn't be the cause of the noise. The unwanted effect of noise in the circuit is called interference. Often, noise cannot be eliminated. The solution to this problem is to reduce their voltage so that interference does not form $[2,10]$.

\section{Materials and methods}

The main methods applied to counter hindrances include shielding, grounding, balancing, filtering, isolation, diversity and orientation, adjustment of the size of the total resistance of the scheme, the choice of cable, suppression (in frequency or time domain). This work is based on the filtering method $[6,7]$. The first thing that needs to be made for receiving reliable information is the restriction of frequency strip where the necessary signalis.

Hydroacoustics is a section of acoustics that studies the radiation, reception, and propagation of sound waves in a real aquatic environment (in the oceans, seas, lakes, etc.) for underwater location and communication. Signals coming to the antennas of submarines and surface ships are accompanied by noise (both of the ships and the environment), and for their effective processing, it is necessary to use a filter amplifier, the development of which is the aim of this work. This device will be placed in the systems of submarines or surface ships.

According to the requirements to the filter amplifier, it must be provided with:

- Galvanically isolated input

- Gain of the amplifier - $40 \mathrm{~dB}$

- Bandwidth of the amplifier at a level of $-3 \mathrm{~dB}$ from 0.5 to $10 \mathrm{kHz}$

- Load resistance $1 \mathrm{kOhm}$, load capacitance $1 \mathrm{nF}$

- Uneven transmission coefficient in the frequency band of not more than $3 \mathrm{~dB}$

- Attenuation at frequencies below $500 \mathrm{~Hz}$ and above $10 \mathrm{kHz}$ not less than $12 \mathrm{~dB} /$ oct.

These requirements are derived from the fact that the information required for processing is represented in a certain frequency band. The lower frequency limit is responsible for the signal range, and the upper limit should be approximately equal to half of the ADC sampling frequency. The unevenness in the frequency band is responsible for the accuracy of processing all signal components. Amplification is necessary for more accurate processing of weak signals. The rolloff from the bottom is determined by the presence of out-of-band interference, the rolloff from the top is related to the ADC sampling rate. The load resistance indicates what load the filter will work with. The output capacitance characterizes the antenna capacitance.

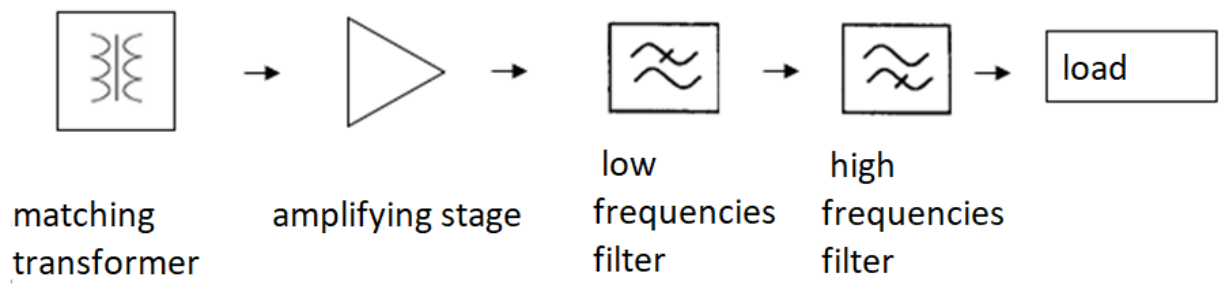

Fig. 1. Block diagram of the filter amplifier.

From the structural diagram of the amplifier shown in Figure 1, we can see that the signal coming from the matching device (transformer in our case) is first amplified, then it is filtered, after which it goes to the load. The load can be both the ADC and its input buffer. Active RC-filters are used for signal filtering, because they have several advantages: 
- Absence of inductors, which ensures manufacturability, reduces the size, increases the accuracy of the amplitude-frequency characteristics (AFC)

- Better selectivity due to the possibility of simple cascading of links and the achievement of higher quality factors

- Compensation of attenuation of useful signals or their amplification.

Since the requirements indicate that attenuation at frequencies below $500 \mathrm{~Hz}$ and above $10 \mathrm{kHz}$ should be at least $12 \mathrm{~dB} /$ oct, filtering links of the second order and higher can be applied. The transfer functions of the filter links can be approximated by Butterworth, Chebyshev, and Bessel polynomials of the 2nd order, which determine the shape of the amplitude-frequency characteristics (AFC), which are presented in Figure 2. Thus, a specific polynomial approximation is selected for the required filter parameters $[3,8,9]$.

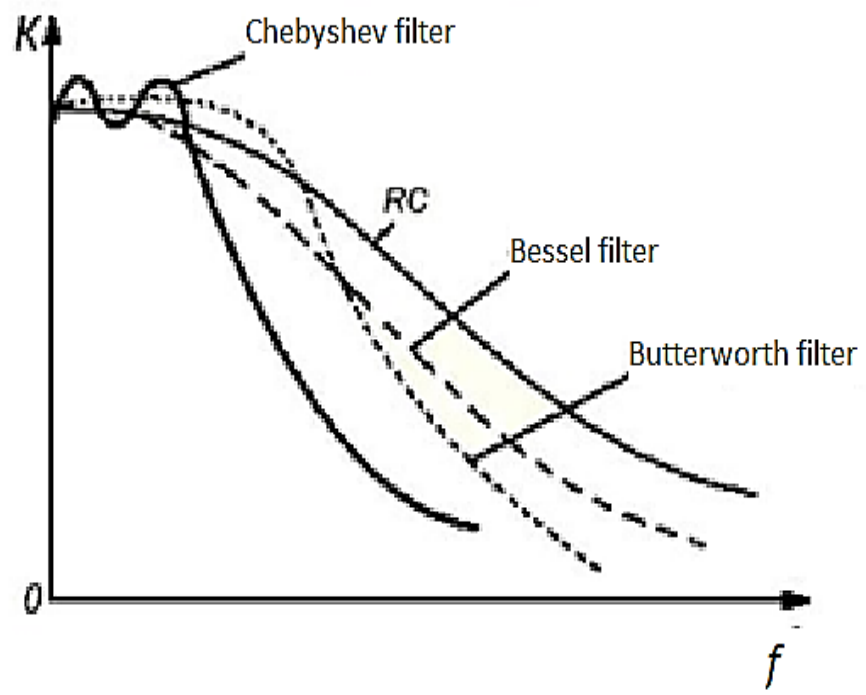

Fig. 2.Frequency response comparison for various second-order polynomials [3].

For the implementation of TK, a Butterworth second-order filter was selected, which describes the frequency response with the flattest part in the passband and a relatively low decay rate. The choice of the implementation circuit of the Butterworth filter is quite wide. The option of representing the circuit in the form of Sallen-Kay units, as the most common type of second-order filters, was chosen. The described circuit is also known as a VCVS filter, a voltage-controlled voltage source. It is sometimes called a "multi-loop feedback filter."

\section{Results}

To analyze the developed circuit shown in Figure 3, the Micro-Cap circuit simulation program is used. It allows to conduct frequency and time analyses, analyses of transients, intrinsic electrical noise and obtain other characteristics of electronic circuits. 


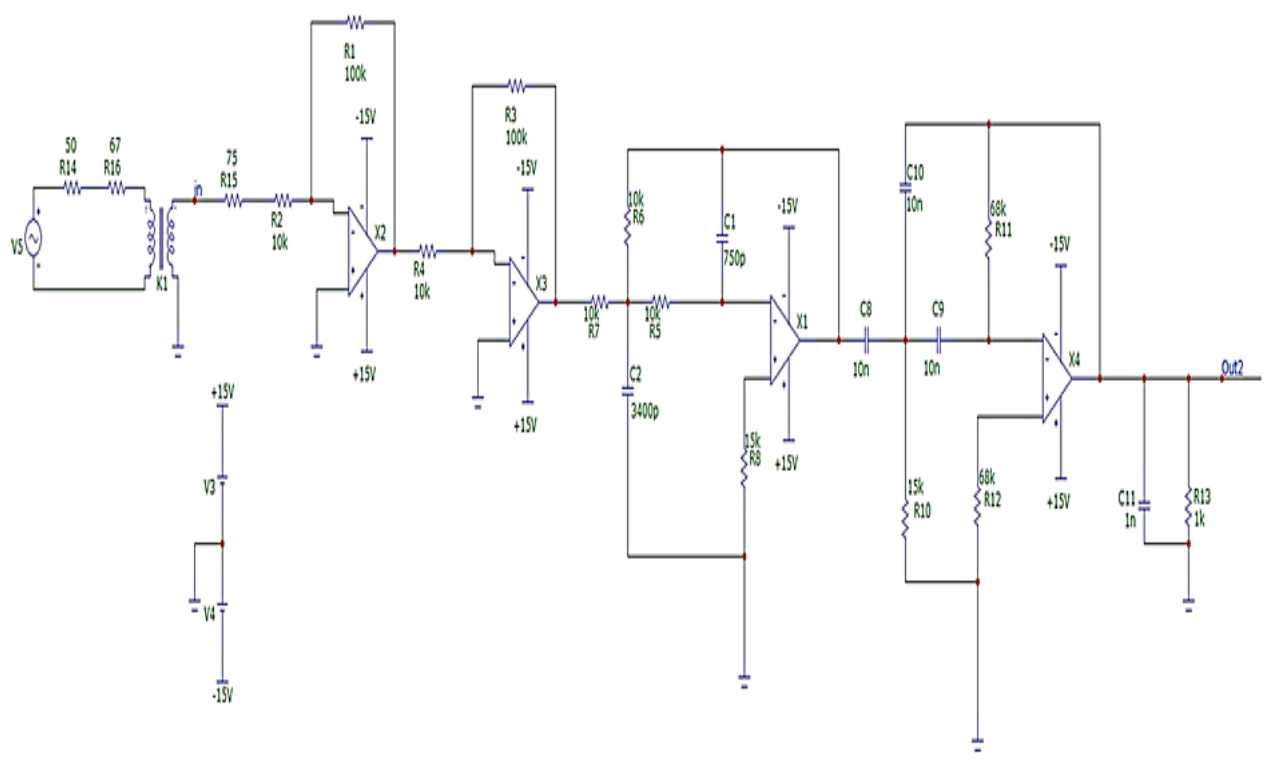

Fig. 3. Schematic diagram of the amplifier channel in the Micro-Cap program.

The output resistance of the signal source is represented by an external resistor R14. Since the transformer model in Micro-Cap does not take into account the resistance of the windings, $\mathrm{R} 15=75 \mathrm{Ohm}$ and $\mathrm{R} 16=67 \mathrm{Ohm}$ resistors are introduced to simulate them. The developed amplifier consists of two amplification stages and two Sallen-Kay filter units. Two identical amplification stages, with amplification factors of 10 each, give a total transfer coefficient of 100. Each amplification stage is constructed according to a circuit with an inverting switch of the op-amp and has negative current feedback. Next is a lowpass filter with a cut-off frequency of $10 \mathrm{kHz}$ and a frequency response slope in the attenuation region of $12 \mathrm{~dB} /$ oct. Then there is a high-pass filter with a cutoff frequency of $0.5 \mathrm{kHz}$ and with a similar slope of the frequency response.

The values of each element of the amplification stages were taken from the most frequently used devices and adjusted for the necessary amplification. The calculation of the elements of the Sullen-Kay links was carried out taking into account the table coefficients of the Butterworth filter: $m_{1}=2,12 ; m_{2}=0,47$.

Since the developed amplifier in real conditions operates on a load receiving a signal through a long cable, R13 and $\mathrm{C} 11$ are set to simulate these operating conditions. The output voltage spectra of power suppliers used in marine applications contain parasitic discrete components which destabilize the performance of amplifiers. To suppress them in practice, RC circuits are placed in the op-amp power circuit, the ratings of which are selected so that the voltage drop across the resistor is acceptable, and the capacitor is selected based on the required parameters for suppressing spurious discrete components. In the first amplification stage, as a rule, an op-amp with a low level of intrinsic electrical noise is installed, because it makes the main contribution to the overall level of intrinsic electrical noise of the entire circuit. In this regard, the intrinsic noise of the op-amp of the subsequent stages can be neglected. When developing an amplification channel in the signal pre-processing equipment of HAC signals, the equivalent antenna capacity is always taken into account, the signal from which is fed to the input of the filter-amplifier.

When developing this scheme, these facts were not taken into account, because the main task was to obtain the amplitude-frequency characteristic that meets the requirements. The resulting frequency response is shown in Figure 4. 


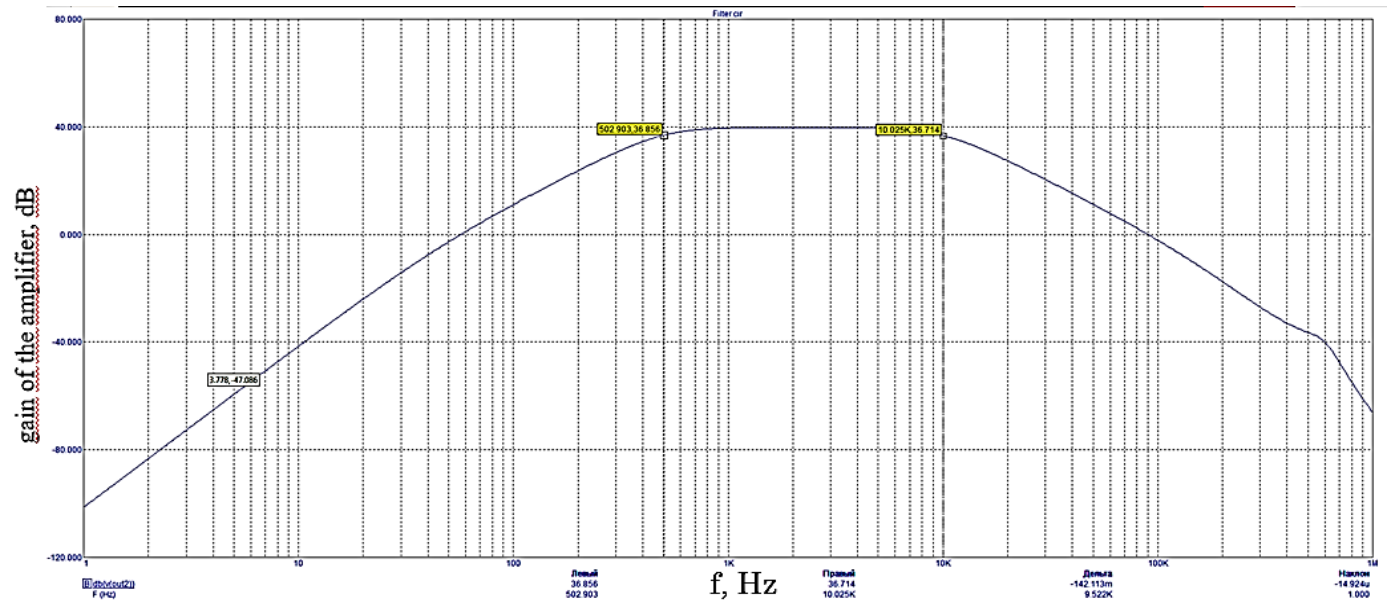

Fig. 4. Frequency response of the developed amplifier channel in the Micro-Cap program.

The characteristic shows that the frequency response meets the requirements of the technical specifications, namely:

- Uneven transmission coefficient in the frequency band of not more than $3 \mathrm{~dB}$

- Attenuation at frequencies below $500 \mathrm{~Hz}$ and above $10 \mathrm{kHz}$ at least $12 \mathrm{~dB} /$ oct

- Amplifier transfer coefficient - $40 \mathrm{~dB}$

- Amplifier bandwidth at a level of $-3 \mathrm{~dB}$ from 0.5 to $10 \mathrm{kHz}$

- Galvanically isolated input - transformer.

\section{Conclusions}

The developed filter meets all the requirements. The circuit was assembled in accordance with the designated structure (Figure 2). During the simulation, an amplitude-frequency characteristic was obtained which meets the conditions.

The developed filter amplifier meets all the stated requirements and can be used in the HAC, namely in modules consisting of several developed amplifiers, which include hermetic devices, i.e., devices operating inside an antenna, submarine noise detectors, or devices with robust hulls.

\section{References}

1. Concern Okeanpribor JSC. - Access mode.

2. J. Keller, J. Papadakis. Wave Propagation and Underwater Acoustics 232 (1980).

3. P. Horowitz, W. Hill. Art of circuitry. (BINOM Publishing House 704, 2014.

4. B.N. Abramovich, A.A. Veprikov, Yu.A. Sychev, D.A. Lyak. TsvetnyeMetally Use of active power transducers in industrial DC power systems supplying electrolysis cells $\mathbf{2}$, 95-100 (2020)

5. C.Savard, A.Nikulina, C. Mécemmène, E. Mokhova. Journal of Open Innovation: Technology, Market, and Complexity The electrification of ships using the Northern Sea Route: An approach 6(1), 13 (2020).

6. A.Nikulin, D. Ikonnikov, PAfanasev. Journal of Applied Science and Engineering Application of individual mobile soundproof cabin at process operator working area 23 (2), 333-340 (2020) 
7. E.D. Kanygina, O.V. Denisova, I.V. Rastvorova. Proceedings of the 2019 IEEE Conference of Russian Young Researchers in Electrical and Electronic Engineering Optical and electrical control in printed circuit board manufacturing 536-538, (2019)

8. M.Carrizosa, N. Stankovic, J. Vannier, Y. Shklyarskiy, A. Bardanov. Journal of Mining institute Multi-terminal dc grid overall control with modular multilevel converters 243, 234-241 (2020).

9. Y.K. Vyboldin, S.V. Borisov. Journal of Physics: Conference Series Noise immunity of signal demodulation procedure in binary coded communication channels with frequency-positioned multiplexing 1384(1), (2019).

10. S.V. Borisov, Y.K. Vyboldin. IOP Conference Series: Earth and Environmental Science Method of estimating linear dimensions of an object by subsurface radar sounding 194 (6), 062005 (2018). 